The father was the donor in ten cases, the mother's half-sister in one and a non-relative in three.

The blood-vessels used were the radial artery in seven cases and a forearm vein in seven cases on the donor; on the baby the femoral vein was used in four instances and the jugular in ten.

The duration of the transfusion varied from five to fifteen minutes. In the case that took fifteen minutes, Case 11, the father fainted, reducing his bloodpressure so low that it took longer to fill the baby with blood.

Table 2 shows the cases that have been reported in the literature. In their essentials they are all exactly like my own cases, details of which are given in Table 1 .

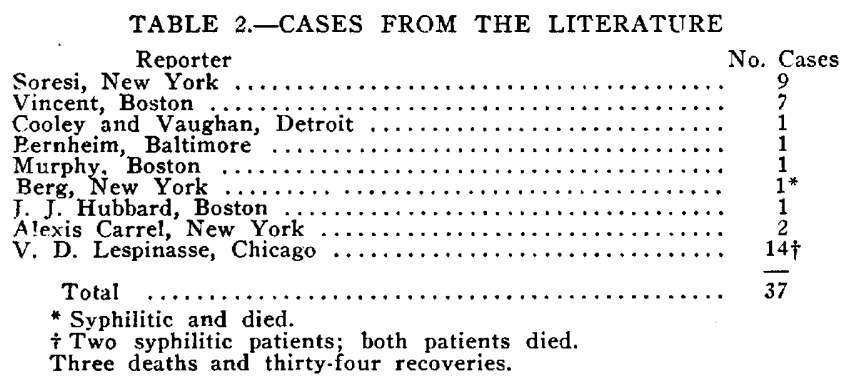

CONCLUSION

Direct transfusion of blood stops the bleeding, restores the lost blood and aids in overcoming infection. Direct transfusion of blood has cured where all other methods have failed. Transfusion should be used early, but so long as there is a spark of life evident, it is never too late to transfuse.

The experimental part of this work was done in the Laboratory of Experimental Surgery of Northwestern University Medical School.

7 West Madison Street.

\section{COMPOUND FRACTURES OF THE BONES OF THE EXTREMITIES *}

W. L. ESTES, M.D., F.A.C.S.

Director and Physician and Surgeon-in-Chief of St. Lule's Hospital SOUTH BETHLEHEM, PA.

This paper may be better followed and understood if at the outset its matter is indicated in the following postulates:

1. In civil practice a compound fracture is always not only a solution of the continuity of a bone, but also a lacerated wound of the soft tissues in continuity from the periostetim to, and including the skin.

2 . Violence necessary to produce a compound fracture of the bone of an extremity must be very great; hence the traumatism is extensive. Commonly the bone is comminuted and the laceration of the soft tissues very severe.

3. Compound fractures are practically always infected wounds.

4. The management of these injuries must include the treatment of a fractured bone and the treatment of a more or less extensive infected lacerated wound of the soft tissues of the same area.

An examination of 2,089 fractures admitted into St. Luke's Hospital shows that 800 of these fractures

\footnotetext{
* Read by invitation before the New York State Meủical Society.
}

were compound. In regions where large manufacturing establishments are located, and also where railroads are many and active, the relative frequency of compound fractures to all fractures is therefore $1: 2.6$, or 38 per cent. of the fractures.

The management of compound fractures of the extremities must resolve itself into two general considerations, namely, (1) the treatment of the general condition of the patient brought about by the injury; (2) the treatment of the injury itself.

A patient's reaction to these severe traumatisms depends on many individual peculiarities, on his environment and on the circumstances of the injury. Individual resistances to psychic shock, to "anociassociation," to loss of blood, must all enter into consideration. The horrors of a severe railroad or factory wreck and the conditions of the locale of the accident have also their effect. In short, a compound fracture, as every other disease or injury does, varies in every case, and should be treated as individual. The treatment must be adapted to the circumstances of each case, not the case adapted to any set method of procedure. This I consider a most important matter and I try to emphasize it on all occasions.

\section{FIRST AID}

The intelligence and skill of a practitioner in many instances, will be taxed to the utmost to meet the necessities of first-aid treatment of compound fractures. One cannot too much emphasize the importance of the proper initial treatment of these injuries. As I have said so often, the fate of the limb and perhaps the life of the patient depend on the manner of the first aid.

First, the general condition of the patient must be gauged as accurately and rapidly as possible. Stimulants may be necessary and should be used (alcohol is not a stimulant and should not be given). An analgesic should be given; as a rule, morphin, in full dose, combined with a small dose of atropin and given hypodermatically, I think, is the best. The control of hemorrhage should be sedulously sought. If practicable to avoid it, a tourniquet of any kind should not be used to control hemorrhage in these cases. If nothing else avails and a tourniquet must be used it ought to be applied some distance away from the seat of fracture. In cases of compound fractures of the bones of the legs, for instance, the tourniquet should be applied to the lower third of the thigh, or if of the bones of the forearm, to the lower third of the arm. An elastic constrictor is best and much safer.

Hemorrhage may as a rule be controlled and at the same time another indication, namely, protecting the wound, may be carried out; by packing the open wound with sterile gauze or any sterile fabric, placing thick compresses of sterile cotton wool or any other clean material over the wound and bandaging it firmly in place, from the extremity, over the wound, and to at least six inches above the fracture. Great care must be used to prevent any further soiling of the wound. No effort should be made to "set the bones," and in no case should a projecting end or fragment of bone be allowed to recede under the skin. unless means are at hand of making adjustment and proper fixation of the bones and limb, and unless the projecting bone and the edges of the external wound have been carefully cleansed and disinfected beforehand. Death has often resulted from the infection 
occasioned by unnecessary manipulation of a wound without previous sterilization of the hands. Never should a finger be thrust into any wound unless the finger and the wound have been previously cleansed and sterilized.

Careful fixation "in the position assumed by the injured limb" should be employed, tunless it is clear that the ends of the fragments are where they will surely do more damage unless their position is changed. The patient should then be transported where he may receive proper permanent dressing and such treatment as his case will require.

The surgeon should try to determine at the very beginning of his responsible management of every case of compound fracture, the following points:

I. What will be the best treatment of this particular injury, considering: (1) the individuality and the physical condition of the patient; (2) the environment of the patient; (3) the actual condition of the injured member.

II. What method of treatment or operation will most likely result in the shortest time of disability of the patient and give him afterward the best functional result?

PERMANENT TREATMENT: I. SPECIAL CONSIDERATIONS

1. Individuality of the Patient.-Before everything clse I would place the consideration of the patient himself. That is to say, I would learn, if possible, not only the actual physical condition of the patient with regard to the tolerance of the trauma, its effects and the extent and degree of the local injury, but also his habits, his temperamental peculiarities and his general health and resistance. Obviously, it would be highly improper to treat an exhausted patient, or a very old person, or a very young child, or a diseased and weak individual, as one would treat a vigorous adult in prime condition.

Thus, after assuring himself that hemorrhage has been thoroughly controlled and that the patient's suffering has been relieved as far as practicable, the surgeon should at once make a thorough physical examination of the patient. He should carefully note all organic lesions, indications of dyscrasias, improper or delayed development, or signs of marked senility. The blood-pressure should always be taken. The immediate subsequent procedures should be determined by this physical examination.

If the patient is in a condition of exhaustion from psychic shock, loss of blood, or "anoci-association," or if his blood-pressure is very low, all extensive and tedious manipulations and operations must be delayed. If he is very old, a long operation with continued anesthesia will be dangerous. Also any method of treatment which will require long confinement to bed should be avoided. Bone repair in most dyscrasic individuals is very slow and always uncertain. Methods must be varied and adapted to meet the indications of the patient's condition.

2. Environment of th. Patient.-The surroundings of the patient will have a very important bearing on the treatment to be employed. When at all practicable, a serious compound-fracture case should be treated in a first-class hospital. It is as a rule far better for the patient to endure the hardship of a one- or two-hour journey in order to reach a hospital than to be subjected to the discomforts and deprivations he would have if he were treated at his home, unless he were very rich. A surgeon is sadly handicapped in trying to treat such cases outside of a well-equipped hospital.

3. The Actual Condition of the Injured Member.As was said in the beginning, a compound fracture is always a complicated injury of very grave severity, and is practically always an infected wound. One must always bear this last fact in mind and treat the case with a full recognition of the importance of thorough cleansing and disinfection of the wound, and especially of thorough drainage.

General anesthesia is usually necessary for the proper examination, handling and dressing of a compound fracture of one of the principal long bones. This is particularly desirable, not so much for the relaxing effect, as to obviate the dreadful pain of the examination and fixation. Compound fractures when not comminuted are much easier to reduce and fix in place than simple fractures.

There is one point in regard to cleansing and disinfecting the injured parts I wish to note, that is, the great value of tincture of iodin for its rapid and very efficient action in disinfecting soiled skin and wounds. One must remember that iodin is of little use over skin which is moist and sodden. In such conditions it will not penetrate but will blister. The surface of the skin to which iodin is applied for disinfecting purposes should be dry. Therefore cleanse the skin of the limb about the wound with benzin, ether or turpentine, and alcohol, then dry carefully and paint it with the tincture of iodin.

Every compound-fracture case will require some operative procedure. In rare instances measures for proper drainage only will be necessary; but in most instances much more will be required.

One must decide at the time of the first permanent dressing :

1. Can the limb be saved?

2. If saved, will the limb be left in a condition for practical use?

3. Will it be best for this patient to run the possible risk of his life, endure a period of long disability, have probably a permanently disabled limb, or will it be best to amputate and thus practically assure his life and shorten the time of his disability by at least one quarter?

1. Conditions Determining Amputation rather than Attempts at Conservation.- $A$. If the skin has been so crushed or lacerated that it is evident that at least three-quarters of the periphery over the fracture will slough, and the muscles beneath are badly lacerated or comminuted, amputation will be inevitable.

$B$. If there has been a circular or annular destructive pressure on the whole periphery of the limb, at the site oi fracture or very near it, amputation will be necessary.

$C$. If in a case of compound fracture with a serious annular laceration of the skin the subjacent muscles are badly comminuted, it will be best to amputate.

$D$. If the injury has been produced by tremendous pressure, as of a car-wheel or heavy pillars of iron or steel, the limb may have the skin of its whole periphery, or nearly all of it killed but not divided, but the muscles beneath will be torn across and the bone comminuted. Such injuries require amputation.

$E$. If the main blood-vessels are torn across in the irregularly jagged way common in these injuries. amputation will be necessary. I do not think anastomoses or transplants of blood-vessels will sttcceed 
in this class of injuries. The laceration of one of the chief vessels when there are two in an extremity does not necessitate amputation. The large nerve trunks will stand much more injury than blood-vessels and may successfully be sutured, unless a long segment of the nerve be destroyed.

$F$. If the bone or bones are comminuted so that the fragments are loose and deprived of periosteum. requiring the loss of as much as $6 \mathrm{~cm}$. (3 inches) of the shaft, this, together with the lacerations of muscles and skin always present in such cases, will require amputation.

2. Probable Final Condition of the Limb.-This is a very important matter and might profitably be considered with the third condition, namely, the best thing for the individual patient. Undoubtedly limbs might be saved which would be quite useless and sometimes positively in the way afterward. Again, for some persons even a useless limb might be considered best if it could be left in a painless condition. A man who has to earn his living by activity and manual labor should be placed in a different category from a man whose livelihood depends on sedentary work requiring very little or no activity. Also for some persons the long confinement and disability resulting from the treatment of a serious compound fracture might be so irksome and indeed disastrous that they might prefer an amputation, and this would be best.

With regard to the danger to life there is no question that conservation is much more hazardous. Analysis shows that in 8.8 per cent. of the cases of compound fractures treated conservatively, the patients died. The average mortality after single major amputation is but 4.54 per cent. The average disability from compound fractures of the femur and both bones of the leg is thirteen months. That of amputation is not quite five months. Of course, this means that the patient may get about, and through the use of an artificial limb may return to a life of comparative activity and much usefulness, though rarely will he be able to return to his former occupation, if it were a laborious one.

Having decided to attempt conservative treatment of the extremity, the first consideration should be to cleanse the wound and surrounding skin. Disinfection (sterilization) of skin and wound should be done thoroughly, especially the ends of the fragments if one or more has penetrated through the skin. Some extension apparatus, such as the Lemon or Lambotte extension bar, is very useful in these cases. Reduction is usually not difficult, on account of the facts that one may see or directly feel the ends of the fragments and guide them by forceps or some kind of lever, and that the muscles are usually so badly lacerated that they do not offer the resistance which is nearly always present in cases of simple fractures.

The fingers should be kept out of the wound as much as possible. I think there can be no doubt that infection is most apt to occur from finger manipulation in the wounds. By a little practice and experience one will learn to perform all the necessary manipulations with the aid of instruments.

Frequently the wound made by the injury, by a little extension or direction, may be used as a way of approach to the bone. The muscles usually are so much lacerated that an incision of the skin and fascia only is necessary. In short, the injury has frequently prepared a way to the bone and if any further incision is necessary it may be so made as to assist in another cardinal object, namely, drainage. One objection to the open method of treating fractures of the long bones of the extremities, that is, the necessary wound, has in compound fractures been removed by the traumatic opening of the tissues.

Direct fixation of the fragments is nearly always best. A rigid splint or plate applied directly to the bone is better than wiring the fragments. In selected cases a nail or nails may be used. I cannot understand the objections and warnings of some otherwise very up-to-date and experienced surgeons against the use of plates in compound fracture cases. My experience and conviction have always been that these are the very cases which demand plates. No valid objection can be offered to sterile fixation instruments unless they in some way harm the fragments. If a proper plate is used in the proper manner it does not injure the bone.

Even if the wound cannot be thoroughly sterilized (and it is exceedingly difficult to do this), suppuration and the evils of infection are diminished locally by a proper metallic plate. Researches at Johns Hopkins Hospital over twenty years ago proved the inhibiting influence of many metals on bacterial growth. Iron and steel are not thus active, as they oxidize too readily, but silver, copper and nickel and their alloys are active inhibitors.

Since 1886 I have used a plate made of Wessel silver, and I have used Wessel silver pegs for many years to fasten this plate to the fragments. I am sure this plate has always seemed to exercise a beneficent effect in compound fractures even with active suppuration present. Besides, fixation relieves pain and further injury which might be caused by the excursions of the ends of the unfixed fragments. After fixing the ends of the fragments one must decide about suturing the lacerated soft tissues. As was said before, blood-vessels are injured in such a way in compound fractures that not only are long stretches absolutely destroyed, but the vasa vasorum are thrombosed for some distance on either side of the evidently destroyed area. The vessel-walls will not bear tension or constriction. I think, therefore, that vascular anastomoses and implantations cannot be used. This is all the more true because these cases are always infected more or less, and vascular anastomoses do not succeed except in sterile fields. Arteries and large veins, therefore, should be ligated and not sutured. Divided nervetrunks may be successfully sutured in these cases and this should be done when necessary. Tendons and muscles may also be sutured, but not when they would seriously impede or block proper drainage. The lacerated fascia and skin should be pared along the margin of the original wound and then be closed with interrupted sutures, sufficiently close to prevent gaping of the wound, but not so as to interfere with free drainage.

Drainage is all important. There will have been very considerable hemorrhage. In very painful lacerated wounds the muscular coats of the local arteries are usually in a condition of almost tetanic spasm. Relief of the pain and complete rest of the limb will be followed by relaxation of the arteries; the result will be that small vessels not perceived during the operation will bleed after the wound is closed. Considerable oozing will always occur. In addition to the oozing blood the opened lymph-spaces will pour out 
quantities of serum. All this fluid should be released and drained out of the wound. Unless this is done, disaster will almost surely occur. The reasons for this are so well known it is not necessary to go further into this.

A very important point with regard to drainage is that it should always be done in such a way and the drains so placed as to avoid all tension and harmful pressure in the wound. This is particularly important when the skin has been torn from the fascia and turned back as a flap, or raised up by a large effusion of blood. Most of the blood-supply has been cut off from the skin in such cases, and any pressure or much tension on the skin will cause a slough. Multiple incisions should be made through the skin and drainage by canalization (without the use of any drain material) should be employed. The incision should be so placed as to assist in relieving tension.

Thus, the bone having been fixed, the lacerated soft tissues sutured, the wound closed and proper drainage provided, a masse dressing of dry, sterile, absorbent material should be applied with fairly firm pressure, and over all a gypsum splint with strips of flexible wood or light metallic strips worked into it, should be molded, and so placed as to give support and some elasticity to the dressing. If all goes well the dressing may remain on for three weeks, then be changed and a similar one applied. Usually all drainage-tubes and gauze packs may be removed and left out at this first dressing. Also it is my custom at this dressing to remove the pegs which hold the plate, unless it be a fracture of the femur. If the wound has not closed over it the plate may also be removed. If the plate is well enclosed and buried by union of the flaps no effort should be made to remove it. As was said before, the plate does not cause any irritation if allowed to remain; on the contrary it seems to inhibit bacterial growth and hastens union of the wound.

PERMANENT TREATMENT: II, AS TO RESULTS

What method of treatment or operation will most likely result in the shortest disability of the patient and give him the best functional result? This determination is meant to apply only when conservative treatment is to be attempted. The indications for amputation have already been given. The question resolves itself therefore, practically, as it is intended to apply it in this instance, into: Will it be best to use direct fixation to the fragments, if so, what kind?

I have recently analyzed fifty-one cases of compound fractures treated in my clinic in St. Luke's Hospital. These cases are taken from the records of the last five years and represent the cases which could be traced and the final results definitely determined. They represent the following classes of compound fractures.

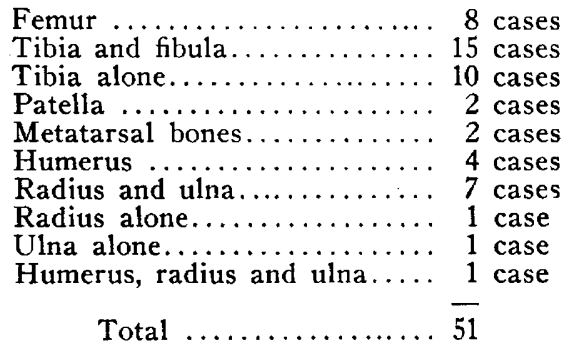

Direct fixation of the fragments was made in 35 cases, of which 28 were plated and 7 were wired. In
13 cases external fixation only was used; 3 patients were moribund when admitted and died within a few hours after admission. (No special treatment and not enumerated further.)

The cases in which plating was done were the following:

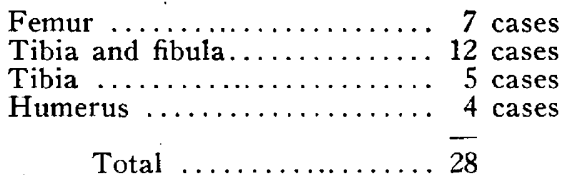

The cases in which wiring was done were the following:

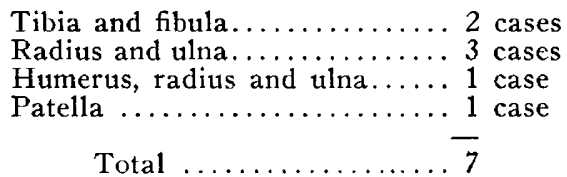

The external fixation apparatus was practically the same in all the cases, namely, a reinforced gypsum splint carefully molded over a large masse dressing of sterile gauze and cotton wool. The average length in days of confinement in bed in the various injuries was:

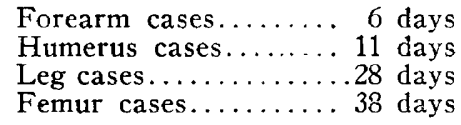

The average time in days spent in the hospital in the various injuries was:

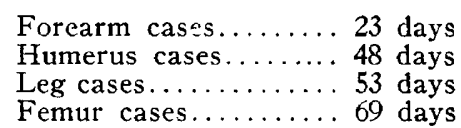

The average length of time the patient was away from work, that is, until he returned to his occupation, was six months in leg fractures and thirteen months in fractures of the femur. For fractures of the upper extremity the time of disability has not yet been thoroughly worked out, but it appears to be about fottr months. All except three of the patients who had direct fixation were operated on within forty-eight hours. Two cases of compound fracture of both bones of the leg were plated on the fifteenth and eighteenth day after injury respectively, because the fragments could not be kept in place. The case of compound fracture of the humerus and both bones of the forearm was wired on the tenth day after injury, also because by other means the fragments could not be kept in apposition.

Though compound fractures should always be regarded as infected wounds, it is noteworthy that it is recorded of these cases that suppuration occurred in only two of those which were fixed by direct application to the bones. One patient with compound fracture of the femur, plated, had a streptococcic infection, but made a good recovery; one patient with compound fracture of the humerus, radius and ulna, wired, had suppuration from a mixed infection, but also made a good recovery. Not one of the patients in the direct fixation cases died.

\section{RESULTS}

In the forty-eight cases treated, there were two deaths, both from septicemia. In one case of compound comminuted fracture of the femur with very extensive injuries to the soft tissues, amputation 
should have been done, but the patient refused amputation and died on the fourth day from acute sepsis. The other case was one of prolonged septicemia from a mixed infection in a man who had a compound fracture of both bones of the leg, for which no direct fixation was done. The mortality was 4.16 per cent.

One patient with compound comminuted fracture of the bones of the leg with dislocation of the ankle-joint had a mixed infection; destruction of the joint and tarsus followed and an amputation became necessary. All but one of the forty-six patients who left the hospital had useful limbs, that is, the one previously noted on whom amputation was done. Of these patients, 40.32 per cent. have returned to their former work and report that they notice no disability.

Roentgenograms which were taken show the conditions of the bones during and after recovery. Roentgenograms in cases of compound fractures are taken (1) after the first permanent dressing; (2) after the fixation plates have been removed; (3) when the union seems complete; (4) after the patient has returned to his occupation.

The number of cases is too small for any set conclusion with regard to the treatment of compound fractures, but certainly in this small series direct fixation has produced much quicker recoveries and far better results than the former conservative methods. It will be noted that not one of the thirty-five patients in whom direct fixation was applied to the fragments died. They all made good recoveries. In the smaller number of cases (thirteen) in which the fragments were not fixed by direct splints on the bones (three hopeless cases are not considered in this reckoning), two patients died of sepsis.

Roentgenograms show that fragments which have been plated together remain in better adjustment than those which have been wired, and the anatomic results are better, except in cases of fracture of the patella. There is less pain after the use of plates than when wire is used, and union results more quickly. It seems. therefore, that compound fractures of the long bones are best treated by rigid splints applied directly to the bones. I believe that the Lane plates are not the best for these cases, however. Experience of other surgeons has shown that in cases of compound fractures the Lane plate must nearly always be removed. Usually it is difficult to get at the screws and then it becomes necessary to use a general anesthetic and to make a considerable additional wound in order to remove this plate; whereas a plate fastened by pegs which proiect through the skin may be released simply by withdrawing the pegs, and if it is necessary to remove the plate this may be done simply by utilizing one or two of the punctures remaining after the peg has been removed, which requires no anesthesia.

A further consideration is the time of disability. My cases show that the average time of disability for compound fractures below the knee was six months and for those above the knee, namely, fractures of the femur, thirteen months. The use of plates thus shortens the time of disability in leg cases by about four months and of the thigh cases, five months. Of the patients, 40.32 per cent. are known to have been able to resume their former occupations. No doubt a number of others were likewise able to do this, but I could find only this number after a year or more from the time their treatment was ended.

8ns Delaware Avenue.

\section{THE ROUTINE TREATMENT OF PUER- PERAL SEPSIS}

JOHN COOKE HIRST, M.D.

Associatc in Obstetrics, University of Pennsylvania, Department of HILADELPHIA

It is obvious that not every case of fever in the puerperium is of septic origin. Engorgement of the breasts, constipation, nervous perturbation, malaria, syphilis, typhoid and other adynamic fevers are some of the many causes of fever entirely separate and distinct from sepsis due to bacterial invasion. It must also be remembered that there are two distinct varieties of sepsis: sapremia due to saprophytic infection and putrefaction of pieces of placenta, membranes. decidua or blood-clot in the uterine cavity, and septicemia due to actual bacterial invasion, most often streptococcic, of the endometrium and adjacent structures, frequently invading directly the blood-current and by far the most dangerous variety.

Sapremia answers promptly to a single evacuation of the uterus and disinfection of the genital canal, with possibly a few supplementary daily intra-uterine douches. Septicemia means a long, hard fight, not inf requently a losing one, against many complications.

It is not my purpose to discuss the various means by which bacteria can be introduced into the genital crinal of a parturient patient, except in one particular. One of the causes spoken of is auto-infection, in which the germs are already present, and no one, except the patient herself, is responsible. This is a comforting thought, but merely means that she is suffering from one infection (gonorrheal, for instance) which reduces her resisting power to a supplementary infection by any pathogenic micro-organisms which may be in the lochia, and is really not auto-infection at all.

\section{DIAGNOSIS}

The diagnosis of sapremia is easy. There is a moderate fever, considerable subinvolution of the uterus and a foul brown or brownish-red discharge. A single evacuation and disinfection of the uterine cavity is usually sufficient.

The diagnosis of septicemia is not so easy, in its early stages. The general symptoms are chills, rapidly rising temperature, rising pulse-rate and a physical depression often out of all proportion to the other symptoms.

The local symptoms, on which the diagnosis usually rests, are a foul discharge (though this symptom may be entirely absent), reddened and edematous labia, a false membrane formation and a subinvoluted, boggy, tender uterus. Pelvic exudate is not often an early symptom, though common enough in the later stages. In .doubtful cases, certain methods of precision may be employed as aids in diagnosis. Leukocyte-counts will usually be higher than in any other form of fever in the puerperium, but in some of the worst cases a low count, showing poor resistance, will be obtained. Cultures taken from the uterine cavity, by the Doederlein tube, or cultures directly from the blood-current, are ofter valuable aids, but unless they are taken with the strictest aseptic technic, and submitted to a competent bacteriologist with adequate facilities for their culture and diagnosis, they become the reverse of precise. 